\title{
Everolimus in combination with rituximab induces complete responses in heavily pretreated diffuse large B-cell lymphoma
}

\author{
Jeffrey A. Barnes, ${ }^{1,5}$ Eric Jacobsen, ${ }^{2,5}$ Yang Feng, ${ }^{2}$ Arnold Freedman, ${ }^{2,5}$ Ephraim P. Hochberg, ${ }^{1,5}$ Ann S. LaCasce, ${ }^{2,5}$ \\ Philippe Armand, ${ }^{2,5}$ Robin Joyce, ${ }^{3,5}$ Aliyah R. Sohani, ${ }^{1,5}$ Scott J. Rodig,, ${ }^{4,5}$ Donna Neuberg, ${ }^{2}$ David C. Fisher, ${ }^{2,5}$ \\ and Jeremy S. Abramson ${ }^{1,5}$
}

\begin{abstract}
${ }^{1}$ Massachusetts General Hospital Cancer Center, ${ }^{2}$ Dana-Farber Cancer Institute, ${ }^{3}$ Beth Israel Deaconess Medical Center, ${ }^{4}$ Brigham and Women's Hospital, ${ }^{5}$ Harvard Medical School, Boston, MA, USA
\end{abstract}

Diffuse large B-cell lymphoma is an aggressive non-Hodgkin's lymphoma without a standard therapy for patients who relapse after or are not eligible for salvage autologous stem cell transplantation. In vitro analysis of lymphoma cell lines has shown that everolimus can inhibit cell cycle progression in vitro and inhibitors of the mammalian target of rapamycin have already demonstrated single-agent activity in relapsed non-Hodgkin's lymphomas including diffuse large B-cell lymphoma, validating mammalian target of rapamycin as a viable therapeutic target. We performed an open label phase II study of everolimus, an inhibitor of mammalian target of rapamycin, in combination with rituximab to examine efficacy and tolerability in patients with relapsed/refractory diffuse large B-cell lymphoma. Eligible patients were treated with everolimus $10 \mathrm{mg}$ by mouth once daily on days 1-28 of a 28-day cycle with rituximab administered weekly during cycle one and then on day one of subsequent cycles. Patients were treated for a total of 12 cycles or until disease progression. The primary end-point was objective response rate, with secondary end-points being toxicity, progression-free survival, duration of response, and overall survival. Twenty-six patients (24 evaluable) were enrolled and had an overall response rate of $38 \%$ [90\% CI (21\%-56\%)] with three complete responses and six partial responses among these 24 patients. The median duration of response among responders was 8.1 months. At a median follow-up of 12 months, the overall survival rate was $37 \%$ [ $90 \%$ CI (20\%-54\%)]. The most common grade 3 to 4 toxicities were neutropenia, anemia, and thrombocytopenia. In conclusion, everolimus in combination with rituximab is well tolerated and demonstrates activity in relapsed diffuse large B-cell lymphoma. Further studies of this combination are warranted. Clinicaltrials.gov identifier: NCT00869999

\section{Introduction}

Diffuse large B-cell lymphoma (DLBCL) is the most common lymphoid malignancy in adults, accounting for approximately 25,000 new cases each year in the United States. High-dose chemotherapy with autologous stem cell transplantation cures a minority of patients with relapsed or refractory disease, ${ }^{1}$ but relapsed DLBCL remains an unmet medical need in patients who relapse after or are ineligible for high-dose chemotherapy due to chemotherapy insensitivity, advanced age, or comorbid disease.

Constitutive activation of the PI3K/AKT pathway and mammalian target of rapamycin (mTOR) signaling has been noted to be a critical event in lymphoma pathogenesis. ${ }^{2}$ Everolimus (RAD001) is an orally bioavailable inhibitor of mTOR. In vitro analysis of DLBCL cell lines has shown that everolimus can inhibit cell cycle progression in vitro by inducing G1 arrest and an associated decrease in the phosphorylation targets of mTOR, p70 s6 kinase and 4-EBP-1, as well as retinoblastoma protein, cyclin $\mathrm{D} 3$ and cyclin $\mathrm{A} .{ }^{3} \mathrm{mTOR}$ inhibitors have already demonstrated single-agent activity in relapsed non-Hodgkin's lymphomas, including DLBCL, vali- dating mTOR as a viable therapeutic target., ${ }^{4,5}$ These agents work primarily through cell cycle arrest, so we hypothesized that combining their cytostatic activity with a cytotoxic agent, such as rituximab, may increase clinical responses. In vitro studies have shown that everolimus and rituximab synergistically induce apoptosis in DLBCL cell lines. ${ }^{3}$

We report here the results of a phase II study of everolimus $10 \mathrm{mg} /$ day in combination with rituximab. The patients enrolled in the study had relapsed after or were ineligible for autologous stem cell transplantation. The standard of care for such patients is undefined.

\section{Design and Methods}

\section{Patients' eligibility}

Patients were eligible if they had previously received therapy and had refractory or relapsed disease. There was no limit on the number of prior therapies. Patients were required to have failed or not have been eligible for autologous stem cell transplantation. Patients were $\geq 18$ years old with histologically confirmed DLBCL, measurable disease, ECOG performance status $\leq 2$, absolute neutrophil count $\geq 1 \times 10^{9} / \mathrm{L}$, platelet count 
$\geq 75 \times 10^{9} / \mathrm{L}$, creatinine $\leq 2.0$ times the upper limit of normal, and aspartate aminotransferase/alanine aminotransferase $\leq 2.5$ times the upper limit of normal. Given known toxicities of everolimus, patients were required to have a fasting serum cholesterol $\leq 300 \mathrm{mg} / \mathrm{dL}$ and fasting triglycerides $\leq 2.5$ times the upper limit of normal. Patients with known leptomeningeal or brain metastases, human immunodeficiency virus infection, severely impaired lung function, defined as diffusing capacity of the lung for carbon monoxide of $<50 \%$, chronic active hepatitis, or prior treatment with an mTOR inhibitor were excluded. This study was conducted in accordance with the Declaration of Helsinki, approved by the institutional review board of participating centers, and registered with clinicaltrials.gov (NTC00869999).

\section{Treatment plan}

Everolimus was administered orally once daily at a dose of $5 \mathrm{mg}$ on days 1 through 14 of cycle 1 . If tolerated, the dose was then increased to $10 \mathrm{mg}$ for days 15 through 28 of cycle 1 . For cycle 2 and beyond, patients continued to receive everolimus at a dose of $10 \mathrm{mg}$ daily continuously. Rituximab, at a dose of $375 \mathrm{mg} / \mathrm{m}^{2}$, was administered intravenously weekly for four doses during cycle 1 , and then on day 1 of cycles 2 through 6 . After cycle 6 , patients could receive an additional 6 months of everolimus monotherapy in the absence of disease progression or unacceptable toxicity.

Response was assessed every two cycles by positron emission tomography/computed tomography during cycles 1 through 6 and every 3 months during the monotherapy phase and interpreted according to the International Harmonization Project criteria. ${ }^{6}$ Toxicity was assessed and graded using the National Cancer Institute Common Terminology Criteria for Adverse Events version 3.0 (CTCAE v3.0). For patients who were unable to tolerate the protocol-specified dosing schedule, dose adjustments were permitted. For patients with hematologic toxicity (neutropenia defined as an absolute neutrophil count $\leq 1 \times 10^{9} / \mathrm{L}$ or thrombocytopenia defined as platelets $\left.\leq 50 \times 10^{9} / \mathrm{L}\right)$ treatment was withheld until recovery to $\leq$ grade 2 and resumed at the initial dose with growth factor support for neutropenia. If cytopenias recurred, treatment was again withheld until recovery to $\leq$ grade 2 and everolimus and rituximab were resumed with the everolimus dose reduced by $50 \%$. For patients with grade 3 non-hematologic toxicity, treatment was withheld until recovery to grade $\leq 1$ then reintroduced with the dose of everolimus reduced to $5 \mathrm{mg} /$ day. Additional dose reductions to $5 \mathrm{mg}$ every third day were allowed. No more than two dose reductions were allowed. There were no rituximab dose reductions. Patients with grade 4 non-hematologic toxicity were removed from the study.

mTOR activity was assessed by immunohistochemical analysis of formalin-fixed, paraffin-embedded tissue samples, when available, using phospo-70 s6 kinase antibodies (1:50) (Cell Signaling Technology, Danvers, MA, USA). The intensity and proportion of tumor cell staining was assessed independently using standard light microscopy by two hematopathologists (ARS and SJR) who were blinded to the response to treatment. A case was considered positive for a given antibody if at least $30 \%$ of tumor cells were positive. The cell of origin was determined by immunophenotyping with stains for CD10, BCL6, and Mum1 using the Hans algorithm as previously reported.?

\section{Study design}

We employed a Simon's two-stage design. The primary end-point was overall response rate. Twenty-five patients were required to distinguish between an unacceptable response rate of $10 \%$ compared to a response rate of $30 \%$ which would be considered promising for further study. Sixteen patients were enrolled in stage one, which required at least two responses to proceed to stage two. We then accrued an additional nine patients on stage two to complete accrual. Five or more out of the 25 patients were needed to respond in order to consider this regimen worthy of further study. The study had $90 \%$ power and a $10 \%$ type 1 error rate.

Overall survival, progression-free survival and duration of response were secondary end-points. Overall survival was defined as the time from registration to death, or last known date of survival. Progression-free survival was defined as the time from registration to progression or death. Duration of response was defined among responders only as the time interval between the date of first confirmed response and the date of disease progression or death. Overall survival, progression-free survival and duration of response curves were calculated using the KaplanMeier method with $90 \%$ confidence intervals calculated using Greenwood's formula.

\section{Results}

\section{Patients' characteristics}

Between July 2009 and June 2010, 26 patients were enrolled. Twenty-five patients initiated treatment, and 24 are included in the efficacy analysis. One patient was removed because of progressive disease prior to receiving any study treatment. The other patient was treated, but the only site of measurable disease was subsequently resected 9 months after study entry and found to be infectious (atypical mycobacterium); this subject is included only in the toxicity analysis. The patients' characteristics at the time of entering the study are listed in Table 1 . Their median age was 65 years (range, 33-87). The median number of prior therapies was four (range, 1-7) with five (21\%) patients having undergone prior autologous stem cell

Table 1. Characteristics at enrollment of eligible and treated patients.

\begin{tabular}{lc} 
& $\mathrm{N}=24$ \\
Mean age (range), years & $65(33,88)$ \\
Age $>60$ years & $14(58 \%)$ \\
\hline Sex, female & $10(42 \%)$ \\
Stage III or IV at relapse & $17(71 \%)$ \\
\hline Elevated lactate dehydrogenase & $16(67 \%)$ \\
$>1$ extra nodal site & $10(42 \%)$ \\
\hline ECOG PS 0-1 & $21(88 \%)$ \\
IPI 3-5 & $14(58 \%)$ \\
\hline Median n. of prior therapies (range) & $4(1,7)$ \\
Median months from diagnosis (range) & $14(4,261)$ \\
\hline Refractory to initial therapy & $13(54 \%)$ \\
Refractory to rituximab & $18(75 \%)$ \\
\hline Prior autologous stem cell transplant & $5(21 \%)$ \\
GCB/Non-GCB/Not known & $14(58 \%) / 6(25 \%) / 4(17 \%)$ \\
\hline
\end{tabular}


transplantation. Thirteen patients (54\%) were refractory to initial therapy and $18(75 \%)$ were refractory to rituximab, defined as progressive disease during or within 6 months of rituximab therapy. All patients had previously received rituximab plus $\mathrm{CHOP}$ or a $\mathrm{CHOP}$-like regimen. Fourteen patients (58\%) had a germinal center immunophenotype, six (25\%) a non-germinal center immunophenotype, and information was not available for four patients $(17 \%)$.

\section{Efficacy}

Twenty-four patients were evaluable for response. The median number of cycles was two (range 1-12) and three $(13 \%)$ subjects received more than six cycles. The overall response rate was 38\% [90\% CI (21\%-56\%)] (Table 2). There were three complete responses and six partial responses. Eight of these nine responders had their best response after two cycles of therapy with one patient with a partial response after two cycles converting to a complete response after four cycles. Six of the nine responders had progressed on their prior chemotherapy, two had relapsed within 3 months of an autologous transplant, and one was more than 2 years from R-CHOP but not eligible for a transplant because of advanced age. The median duration of response was 8.1 months (Figure 1). The median progression-free survival was 2.9 months [90\% CI (1.83.8)] (Figure 2A). At a median follow up of 12 months, the progression-free survival rate was $22 \%$ [90\% CI $(8 \%$ $37 \%)$. Fifteen subjects had died by the time of the last follow-up. The median overall survival was 8.6 months $[90 \%$ CI (4.9-16.3)]. At a median follow up of 12 months, the

Table 2. Efficacy.

\begin{tabular}{lc}
\hline Best response & $\mathbb{N}=24(\%)$ \\
Overall response & $9(38 \%)[90 \% \mathrm{CI}(21,56)]$ \\
Complete response & $3(13 \%)$ \\
Partial response & $6(25 \%)$ \\
Stable disease & $2(8 \%)$ \\
\hline Progressive disease & $13(54 \%)$ \\
\hline
\end{tabular}

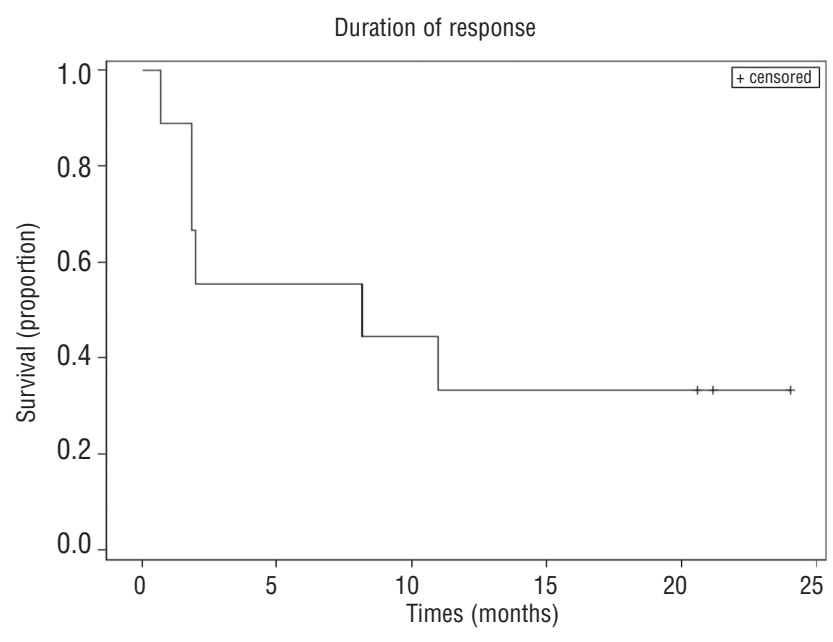

Figure 1. Kaplan-Meier curve of the duration of response for the nine responding patients. overall survival rate was 37\% [90\% CI (20\%, 54\%)] (Figure 2B).

All three patients with a complete response are alive and free of disease (Figure 3). One patient was removed from the study after five cycles of treatment and consolidated with an allogeneic stem cell transplant. One patient with progressive disease after salvage chemotherapy who then underwent allogeneic transplantation without a response began everolimus on day 174 after the transplant and had a complete response to study treatment after two cycles. This patient then went on to have consolidation therapy with three donor lymphocyte infusions (the first 118 days after starting everolimus) and remains disease-free. One patient completed 12 cycles of everolimus and is diseasefree 12 months after completion of therapy. Of the six patients with a partial response, three are alive. Three patients received additional therapy after progression including two who had allogeneic transplants and remain alive. One patient in a partial response after two cycles died in a motor vehicle accident. Two patients died of progressive lymphoma.

The immunophenotype of the cell of origin was available for 20 of the 25 patients with no clear pattern of response among those with germinal cell B-cell (GCB) and non-GCB subtypes (Table $3 \mathrm{~A})$. There was also no correlation with International Prognostic Index score at relapse and with

A

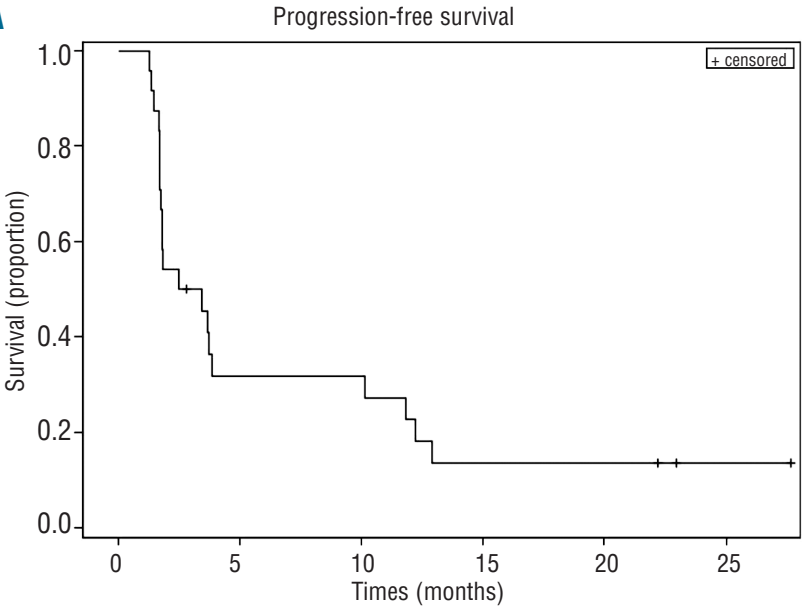

B

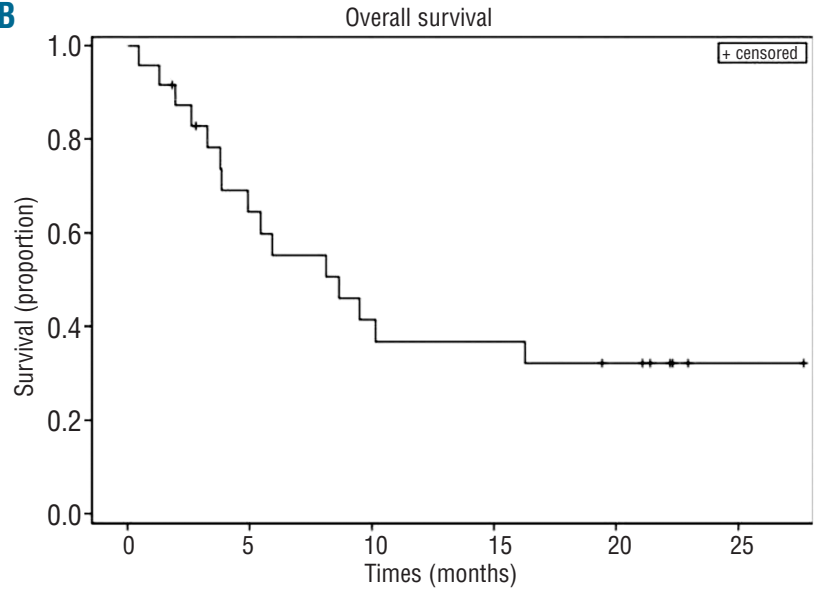

Figure 2. Progression-free (A) and overall survival (B) for the 24 evaluable patients. 
response. The phospho-70 s6 kinase immunophenotypic marker of mTOR pathway activation was available for 13 patients (Table 3B), 11 from the pretreatment specimens and two at the time of relapse. One patient had biopsies available both pre-treatment and at relapse; there was no mTor activation at either time point. All patients with a complete response had evidence of mTOR activation, but six of eight non-responders also had evidence of mTOR activation with no difference seen between the two groups.

\section{Safety and tolerability}

The most common grade 3 to 4 toxicities were neutropenia, anemia and thrombocytopenia (Table 4). Eleven patients had treatment withheld because of toxicity ( 6 for neutropenia, 3 for thrombocytopenia, and 2 for nausea). Only one patient did not achieve dose escalation of everolimus from $5 \mathrm{mg}$ to $10 \mathrm{mg}$ during cycle 1 because of thrombocytopenia. Four patients required dose reductions

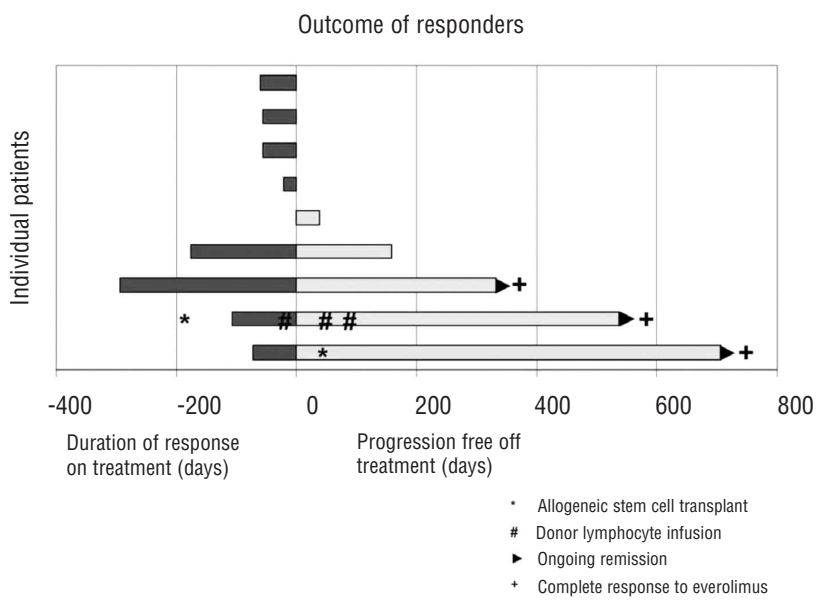

Figure 3. Outomes of the nine responders. Dark bars denote response duration on therapy to day 0 , the first day off treatment. Clear bars then demonstrate progression-free survival after treatment. The three patients with ongoing remissions at last follow up are indicated with a $(\downarrow)$ symbol. The three patients with a compete response to everolimus are indicated with a $(+)$ symbol. One patient received an allogeneic stem cell transplant, indicated by a $\left({ }^{*}\right)$ symbol, prior to starting everolimus and also received one donor lymphocyte infusion (\#) during everolimus treatment and two additional donor lymphocyte infusions after completing everolimus.

Table 3. Response assessment by cell of origin and activation of the mTOR pathway.

\section{A. Response by cell of origin}

\begin{tabular}{lcc} 
& Responders & Non-Responders \\
GCB & 6 & 8 \\
Non-GCB & 2 & 4 \\
\hline Cell of origin N/A & 1 & 4 \\
\hline
\end{tabular}

\section{B. Response by activation of mTOR pathway}

\begin{tabular}{lcc} 
& Responders & Non-Responders \\
pS6 + & 4 & 6 \\
pS6- & 1 & 2 \\
\hline N/A & 4 & 8 \\
\hline
\end{tabular}

GCB: Germinal center B cell; pS6: phsophoS6 kinase; N/A: tissue not available. to $5 \mathrm{mg}$ daily ( 2 due to neutropenia, 1 due to thrombocytopenia, and 1 due to fatigue and nausea). Disease progression was the most common cause of discontinuation of therapy, with 18 (72\%) patients stopping therapy because of progression. One patient stopped therapy after eight cycles because of toxicity (pneumonitis), one stopped due to the investigator's decision, and one patient died in an automobile accident while in partial remission. One patient completed a full year of everolimus therapy. One patient died of hepatitis B reactivation after removal from the study because of disease progression. This patient was positive for hepatitis surface antibody, negative for surface antigen negative, and had undetectable viral DNA prior to study entry. This patient had hepatitis B reactivation 3 months after the final study treatment and after additional rituximab-containing salvage therapy.

\section{Discussion}

Relapsed and refractory DLBCL remains an unmet medical need given the low rate of cure in patients relapsing after R-CHOP chemotherapy. ${ }^{1}$ The rationale for everolimus in DLBCL is based on preclinical data showing dependence on the mTOR/PI3kinase pathway. ${ }^{2}$ In a phase II study of everolimus monotherapy in 77 patients with relapsed non-Hodgkin's lymphoma, 47 had DLBCL, among whom the overall response rate was $30 \%$, with no

Table 4. Toxicities (adverse events considered at least possibly related to study treatment).

Treatment-related toxicities by grade
Adverse Event Grade 2 Grade 3 Grade 4 Grade 5 Grade 3-4 (\%)

General

Fatigue

Anorexia

Dry mouth

Mucositis

Rash

$\begin{array}{lllll}6 & 2 & 0 & 0 & 8\end{array}$

Hematologic

Neutropenia

Anemia

Thrombocytopenia

Leukocytosis

Gastrointestinal

Constipation

Diarrhea

Dysphagia

Viral hepatitis

Metabolic

Hyperglycemi

Hypokalemia

Hypophosphatemia

Hypertriglyceridemia 4

Hyperuricemia

Infection

Infection with

grade 3 or 4 neutropenia

Infection with 0

$<$ grade 3 neutropenia

Pulmonary

Dyspnea

Pneumonitis 
complete responses. ${ }^{4}$ The median progression-free survival and duration of response for the entire cohort were 3.0 and 5.7 months, respectively. In our study of the combination of everolimus and rituximab, we found an encouraging overall response rate of $38 \%$. While the value of the addition of rituximab to everolimus cannot be ascertained from the current study, the combination produced three complete responses, and two patients used protocol therapy as a bridge to allogeneic stem cell transplantation, both of whom are alive and free of disease at a median of 19 months (range, 17-24) (Figure 3). Among patients who did not go on to stem cell transplantation, two patients achieved long-term disease control following everolimus/rituximab treatment (one completing 12 months of therapy in a complete response and one stopping after 9 months due to pneumonitis in a partial response with progression 5 months later).

Our response rate of $38 \%$ in the context of a heavily pre-treated DLBCL population is particularly encouraging given that more than half of the patients were refractory to initial treatment with $\mathrm{R}-\mathrm{CHOP}$ or $\mathrm{R}-\mathrm{CHOP}-$ like regimens, and $75 \%$ were considered rituximab-refractory at the time of enrollment. There are limited data on rituximab monotherapy in relapsed/refractory patients. A Japanese phase II trial reported a $35 \%$ overall response rate to rituximab monotherapy, but all patients were rituximab-naive and the majority had received only one or two prior regimens, while the majority of our patients were rituximab-refractory with a median of four prior regimens. ${ }^{8}$ Accordingly, we expect the response rate to rituximab monotherapy in our population to be quite low.

The combination of everolimus and rituximab was well tolerated. Adverse events were similar to and not increased compared to those seen in studies of everolimus monotherapy. Unlike previous trials of everolimus, clinically significant hyperglycemia and hyperlipidemia were uncommon in our study, and only a minority of patients required dose reductions for toxicity.

DLBCL constitutes a heterogeneous group of diseases with diverse biology and outcomes with modern therapy. Given the heterogeneity, investigation of novel agents must also seek prospective biomarkers predictive of response. In addition to predicting response to initial anthracycline and rituximab-based therapy, the cell of origin may also be predictive of response in relapsed/refractory DLBCL. For instance, lenalidomide was shown to produce a higher response rate in relapsed/refractory DLBCL of non-germinal center origin as assessed by the Hans immunohistochemical algorithm. ${ }^{9}$ As shown in Table 3, we found that cell of origin did not predict response in this small sample.

The level of phosphorylation of the 70 S6 kinase has been shown to predict response to the mTOR inhibitor temsirolimus in renal cell carcinoma, ${ }^{10}$ but we found no correlation with level of activation of the mTOR/PI3kinase pathway in our exploratory analysis. This analysis was performed on original diagnostic biopsy tissue, when available, and the time of fixation was not, therefore, controlled. Since most excisional biopsies of lymphoma sit outside of formalin for several minutes while the tissue is triaged for lymphoma work-up it is possible that this pre-analytic variable known to affect phosphorylation markers resulted in false negatives and may have affected our results.

In summary, we report that everolimus plus rituximab induces responses in heavily pre-treated patients with DLBCL and may serve as a bridge to allogeneic stem cell transplantation. Studies examining the role of mTOR partners', raptor and rictor, in predicting responders are planned for the future. ${ }^{11}$ Clinical studies are also underway evaluating mTOR-directed therapy earlier in the course of this disease and in combination with additional agents to overcome resistance thought to occur through escape via the AKT pathway. These studies are likely to identify responders to this therapy and to improve outcomes for patients with resistant DLBCL.

\section{Funding}

This work was funded in part by Novartis, Inc.

\section{Authorship and Disclosures}

Information on authorship, contributions, and financial \& other disclosures was provided by the authors and is available with the online version of this article at www. haematologica.org.

\section{References}

1. Gisselbrecht C, Glass B, Mounier N, Singh Gill D, Linch DC, Trneny M, et al. Salvage regimens with autologous transplantation for relapsed large B-cell lymphoma in the rituximab era. J Clin Oncol. 2010;28(27):4184-90.

2. Panwalkar A, Verstovsek S, Giles FJ. Mammalian target of rapamycin inhibition as therapy for hematologic malignancies. Cancer. 2004;100(4):657-66.

3. Wanner K, Hipp S, Oelsner M, Ringshausen I, Bogner C, Peschel C, et al. Mammalian target of rapamycin inhibition induces cell cycle arrest in diffuse large B cell lymphoma (DLBCL) cells and sensitises DLBCL cells to rituximab. $\mathrm{Br} \mathrm{J}$ Haematol. 2006;134(5):475-84

4. Witzig TE, Reeder CB, LaPlant BR, Gupta M, Johnston PB, Micallef IN, et al. A phase
II trial of the oral mTOR inhibitor everolimus in relapsed aggressive lymphoma. Leukemia. 2011;25(2):341-7.

5. Smith SM, van Besien $K$, Karrison $T$, Dancey J, McLaughlin P, Younes A, et al. Temsirolimus has activity in non-mantle cell non-Hodgkin's lymphoma subtypes: the University of Chicago phase II consortium. J Clin Oncol. 2010; 28(31):4740-6.

6. Cheson BD, Pfistner B, Juweid ME, Gascoyne RD, Specht L, Horning SJ, et al. Revised response criteria for malignant lymphoma. J Clin Oncol. 2007;25(5):57986.

7. Hans CP, Weisenburger DD, Greiner TC, Gascoyne RD, Delabie J, Ott G, et al Confirmation of the molecular classification of diffuse large B-cell lymphoma by immunohistochemistry using a tissue microarray. Blood. 2004;103(1):275-82

8. Tobinai K, Igarashi T, Itoh K, Kobayashi Y, Taniwaki M, Ogura M, et al. Japanese mul- ticenter phase II and pharmacokinetic study of rituximab in relapsed or refractory patients with aggressive B-cell lymphoma. Ann Oncol. 2004;15(5):821-30.

9. Hernandez-Ilizaliturri FJ, Deeb G, Zinzani PL, Pileri SA, Malik F, Macon WR, et al Higher response to lenalidomide in relapsed/refractory diffuse large B-cell lymphoma in nongerminal center B-celllike than in germinal center B-cell-like phenotype. Cancer. 2011;117(22):5058-66.

10. Cho D, Signoretti S, Dabora S, Regan M Seeley A, Mariotti M, et al. Potential histologic and molecular predictors of response to temsirolimus in patients with advanced renal cell carcinoma. Clin Genitourin Cancer. 2007;5(6):379-85.

11. Sebestyen A, Sticz TB, Mark A, Hajdu M Timar B, Nemes K, et al. Activity and complexes of mTOR in diffuse large B-cell lymphomas-a tissue microarray study. Mod Pathol. 2012;25(12):1623-8. 